\title{
Guest editorial: special section on regression testing
}

\author{
Shin Yoo $\cdot$ Per Runeson
}

Published online: 5 October 2014

(C) Springer Science+Business Media New York 2014

This issue of the Software Quality Journal contains a special section on Regression testing. Regression testing used to be considered as a special type of testing activity that is performed to prevent regression faults, i.e. damages to existing features, introduced by recent modifications to the software. This was a gigantic task involving an infeasible number of test cases, and so research has focused on how to optimise the test suites for a long time.

However, in the days of agile and globally distributed development, cloud computing, and app markets, software is continuously evolving at such a lightning pace that, really, regression testing cannot be left out as a separate activity at the end of testing. The concept of regression faults and test suite optimisation should permeate testing activities of all kinds at all layers.

The papers in the special section reflect the wide spectrum of domains and technical granularities that regression testing can be considered in. The paper "Influences on Regression Testing Strategies in Agile Software Development Environments" considers the influences of regression testing techniques on agile development on a strategic level. It provides insights into industry practice and needs on regression testing, based on three industrial experience reports. The paper "Identifying the Effects of Modifications as Data Dependencies" analyses the precise impact of modifications for more effective test optimisation. We believe that the diversification in the angles from which to consider regression testing will be the key to the wider adoption of the techniques we have developed so far and will develop in the future.

\footnotetext{
S. Yoo $(\square)$

Department of Computer Science, University College London, Gower Street, London WC1E 6BT, UK e-mail: shin.yoo@ucl.ac.uk

P. Runeson

Department of Computer Science, Lund University, Box 118, SE-221 00 Lund, Sweden

e-mail: per.runeson@cs.lth.se
} 